\title{
GROUPS WITH NORMAL SUBGROUPS POSSESSING SUBNORMAL COMPLEMENTS
}

\author{
K. H. TOH
}

\begin{abstract}
J. Wiegold has characterized groups in which every normal subgroup is a direct factor as the restricted direct products of simple groups. In this paper, it is proved that for a group $G$ to have the structure above, it is sufficient that every normal subgroup of $G$ has a subnormal complement in $G$.
\end{abstract}

1. Introduction. A subgroup $A$ of a group $G$ is said to be complemented in $G$ if there is a subgroup $B$ of $G$ such that $G=A B$ and $A \cap B=1$. The subgroup $B$ is called a complement of $A$ in $G$. Adopting a notation of C. Christensen [2], we shall call $G$ an $n D$-group ( $n S$-group, $n C$-group) if every normal subgroup of $G$ has a normal complement (a subnormal complement, some complement). It is clear that

$$
[n D] \subseteq[n S] \subseteq[n C]
$$

where [X] denotes the class of $X$-groups. J. Wiegold [5] has characterized $n D$-groups as the (restricted) direct products of simple groups. The much wider class of $n C$-groups has been studied by C. Christensen ([2], [3]), S. N. Cernikov [1], N. T. Dinerstein [4] and D. I. Zaicev [6]. Our purpose is to show that the classes $[n D]$ and $[n S]$ are identical, i.e. we shall prove the following

THEOREM. Every $n S$-group is an $n D$-group.

The proof employs Wiegold's result cited above and a theorem of Cernikov [1, Theorem 13, Corollary 2] which states that a locally nilpotent $n C$-group is a (restricted) direct product of cyclic groups of prime orders.

2. Lemmas. In all that follows, by the direct product of a set of groups we shall mean the restricted direct product. The symbol $N \triangleleft G$ will denote

Received by the editors March 8, 1973.

AMS (MOS) subject classifications (1970). Primary 20F15, 20F30; Secondary 20D35, 20 D40.

Key words and phrases. Complement, subnormal subgroup, subnormal complement, restricted direct product, simple group, locally nilpotent group, normal closure, minimal normal subgroup.

(C) American Mathematical Society 1973 
the fact that $N$ is a normal subgroup of $G$. For any two elements $a$ and $b$ of $G,[a, b]$ will denote the element $a^{-1} b^{-1} a b$.

Lemma 1. Let $G$ be an $n S$-group, $A \triangleleft G$ and $B$ a complement of $A$ in $G$. Then $B$ is an $n S$-group.

Proof. Since $G=A B$ and $A \cap B=1, B \simeq G / A$. Let $N$ be a normal subgroup of $G$ containing $A$ and $K$ a subnormal complement of $N$. Then in the factor group $G|A, K A| A$ is a subnormal complement of $N / A$. Thus, $G / A$, and hence $B$, is an $n S$-group.

Lemma 2. Let $G$ be a group, $K$ a subnormal subgroup of $G$ and $M a$ nonabelian minimal normal subgroup of $K$. Then $M^{G}$ (the normal closure of $M$ in $G$ ) is a minimal normal subgroup of $G$.

3. Proof of the theorem. Let $G$ be an $n S$-group. Denote by $A$ the subgroup generated by the nonabelian minimal normal subgroups of $G$ and by $B$ that generated by the abelian subnormal subgroups of $G$. Then $A \triangleleft G$, and since conjugates of subnormal subgroups are subnormal, we also have $B \triangleleft G$.

We shall prove that $G=A B$. Suppose, on the contrary, that $G \neq A B$. Then, $G$ possesses a subnormal subgroup $C$ such that $G=A B C, A B \cap C=1$ and $C \neq 1$. Let $c \in C, c \neq 1$, and let $N$ be a normal subgroup of $C$ maximal with respect to the property that $c \notin N$. Since, by Lemma $1, C$ is an $n S-$ group, there is a subnormal subgroup $D$ of $C$ such that $C=N D$ and $N \cap D=1$, and it follows that $D \simeq C / N$. Now by the choice of $N$, every nontrivial normal subgroup of $C / N$ contains the element $c N$. Hence $C / N$, and therefore $D$, possesses a unique minimal normal subgroup. Let the minimal normal subgroup of $D$ be $M$. Then $M \neq 1$ and $M$ is subnormal in $G$. If $M$ is abelian, we have $M \subseteq B$. On the other hand, if $M$ is nonabelian, then by Lemma $2, M^{G}$ is minimal normal in $G$, and so $M \subseteq M^{G} \subseteq A$. In either case, $A B \cap C \neq 1$, a contradiction. Hence $G=A B$.

Next we observe that $A$ is a direct product of minimal normal subgroups of $G$, and $A \cap B \triangleleft G$. It follows easily that $A=A_{1} \times(A \cap B)$ where $A_{1}$ is a direct product of some minimal normal subgroups $M_{\lambda}(\lambda \in \Lambda)$ of $G$. We write $A_{1}=\prod_{\lambda \in \Lambda}^{\times} M_{\lambda}$. Hence $G=A B=A_{1} B$, and since $A_{1} \cap B \subseteq A_{1} \cap$ $(A \cap B)=1$, we have

$$
G=A_{1} \times B=\left(\prod_{\lambda \in \Lambda}^{\times} M_{\lambda}\right) \times B .
$$

Since a normal subgroup of a direct factor of $G$ is normal in $G$, each $M_{\lambda}$ is simple. 
Finally, the subgroup $B$ is locally nilpotent (from its definition) and is an $n S$-group, by Lemma 1 . The result then follows from Cernikov's theorem cited above.

\section{REFERENCES}

1. S. N. Cernikov, Groups with systems of complemented subgroups, Mat. Sb. 35 (77) (1954), 93-128; English transl., Amer. Math. Soc. Transl. (2) 17 (1961), 117-152. MR 16, 565; 23 \#A1714.

2. C. Christensen, Complementation in groups, Math. Z. 84 (1964), 52-69. MR 29 \#1263.

3. - Groups with complemented normal subgroups, J. London Math. Soc. 42 (1967), 208-216. MR 34 \#7648.

4. N. T. Dinerstein, Finiteness conditions in groups with systems of complemented subgroups, Math. Z. 106 (1968), 321-326. MR 38 \#3340.

5. J. Wiegold, On direct factors in groups, J. London Math. Soc. 35 (1960), 310-320. MR 24 \#A164.

6. D. I. Zaicev, On normally factorizable groups, Dokl. Akad. Nauk SSSR 197 (1971), 1007-1009 = Soviet Math. Dokl. 12 (1971), 601-604. MR 44 \#2820.

Department of Mathematics, University of Malaya, Kuala Lumpur, Malaysia 